\title{
Optical burst switching with large switching overhead
}

\author{
C. Y. $\mathrm{Li}^{*}, \mathrm{G} . \mathrm{M} . \mathrm{Li}^{\dagger}$, P. K. A. Wai*, and V. O. K. $\mathrm{Li}^{\dagger}$ \\ *Photonics Research Center and Department of Electronic and Information Engineering, \\ The Hong Kong Polytechnic University, Hong Kong, China \\ Tel: +852 2766-4094, Fax: +852 2362-8439, E-mails: \{enli, enwai\}@ polyu.edu.hk \\ $\dagger$ Department of Electrical and Electronic Engineering, The University of Hong Kong, Hong Kong, China \\ Tel: +852 2857-8425, Fax: +852 2559-8738, E-mails: \{gmli, vli\}@eee.hku.hk
}

\begin{abstract}
The optical burst switching (OBS) schemes to date assume that the switching overhead at intermediate nodes is either negligible or can be considered as part of the processing delay of the control packet. In this paper, we will show that the switching overhead can have a significant impact on the performance of OBS. We have also proposed methods to alleviate the problem.
\end{abstract}

\section{INTRODUCTION}

Optical burst switching (OBS) has been gaining popularity in recent years because OBS can be implemented with current technology. OBS networks use one-way reservation to reserve the required resources for data transmission [1]-[4]. When a packet arrives at a node, it is stored in an electronic buffer until the number of packets with the same destination reaches a threshold value or the first packet in the batch exceeds the storage time limit. The node then sends out a control packet to the destination. After an offset time, a data burst containing the new packets are sent out following the path of the control packet. The control packet reserves the resources at the nodes on the path for the data burst. No acknowledgment is sent back to minimize electronic buffering and the delay time of the data burst. If the reservation by the control packet is successful, the data burst will pass through all nodes on the path from source to destination without any processing and optical-toelectrical (O/E) conversion. No optical buffer is therefore required with OBS. The one way reservation in OBS reduces the complexity of signaling between nodes and also shortens the waiting time of the data bursts at the source nodes. We can implement various routing services such as priority routing by adjusting the offset time between the transmissions of control packet and data burst. Compared to other proposed optical packet switching methods, OBS is more feasible. Unlike the connection-oriented wavelength-routed optical networks, the large propagation delay between nodes is no longer a major system performance concern in OBS. Testbeds of OBS using technology similar to wavelength division multiplexing have been demonstrated recently [3], [4]. While these testbeds demonstrate the feasibility of OBS, i.e., even slow optical

This research is supported in part by the Areas of Excellence Scheme established under the University Grants Committee of the Hong Kong Special Administrative Region, China (Project No. AoE/E-01/99). Additional support is provided by a grant from The Hong Kong Polytechnic University (Project Number A-PF98). switches can be used, they also reveal operation requirements for OBS that have been overlooked before.

The basic requirement of the OBS reservation is that the offset time must be larger than the total processing delay of the control packet at the intermediate nodes. To the best of our knowledge, the effect of the reconfiguration time of the optical switches at the intermediate nodes has not been considered separately from the control packet processing time in OBS performance evaluations. This is equivalent to assuming that either the switch reconfiguration time is negligible, or that it can be treated as a part of the processing delay of the control packet [2]. Currently, fast optical switches which have switching time in nanoseconds or even picoseconds range are only available in small sizes such as $2 \times 2$. Large optical switches (over 100 ports) normally use technologies such as micro-electro-mechanical system (MEMS) that require reconfiguration time of milliseconds [5], [6]. Owing to the rapid increase of optical fiber transmission rate-up to hundreds of gigabits per second-and the necessity to keep a reasonable data burst size, the overhead due to optical switch reconfiguration is no longer negligible. Treating the switch reconfiguration time as a part of the control packet processing delay is simply inappropriate. In fact, a data burst can be transmitted by the optical switch of a node at the same time the node is processing a control packet. A data burst, however, has to be dropped if it wants to go through the switch while the switch is reconfigurating itself. All OBS schemes that treat switch reconfiguration time as a part of the control packet processing delay overestimate the system performance of OBS when the switching overhead becomes large.

In this paper, we investigate the effect of large switch reconfiguration overhead on the performance of OBS schemes. Unlike other proposed optical packet-switching architectures, it is possible for OBS to determine the required switch configurations before a packet arrives at the node using the scheduling information from the control packets. We can therefore use the prior information of the status of the switch to reduce the negative impact of large switching overhead. In Section II, we review typical OBS node architectures, and investigate the performance degradation caused by the switching overhead. We discuss how to make use of the the switching architectures to alleviate the impact of large switching overhead in Section III. The main idea is to take advantage of the fast switching of wavelength converter [7], [8] and the prior 


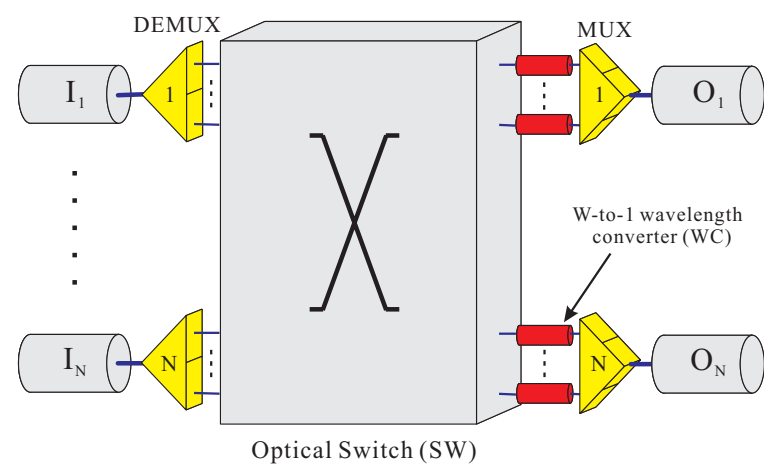

Fig. 1. A typical bufferless $N \times N$ OBS node. From left to right are the wavelength demultiplexer (DEMUX), internal non-blocking optical switch (SW), $W$-to-1 wavelength converters (WCs), and wavelength multiplexer (MUX). The control packet processing unit is omitted.

knowledge of the switch status to decouple the internal path setup/tear down sequences in the optical switch of an OBS node. From the simulation results shown in Section IV, we find that the proposed schemes can significantly improve the OBS performance when the switching overhead is large. We conclude in Section V.

\section{OBS WITH LARGE SWITCHING OVERHEAD}

\section{A. General OBS node architectures}

Most OBS schemes to date assume that a data burst entering from a wavelength channel at any input port of a node is able to switch to any wavelength channel at any output port provided that the output channel is idle. It requires the node to have full wavelength conversion capability and an internally non-blocking optical switch. Two typical bufferless $N \times N$ OBS node is shown in Figs. 1 and 2. The control packet processing unit is omitted for simplicity. We assume that there are $W$ wavelength channels in each fiber. In Fig. 1, an incoming optical signal is first wavelength-demultiplexed by the demultiplexer (DEMUX) into $W$ optical signals at different wavelengths, which is then sent to one of the input ports of the $N W \times N W$ internal non-blocking optical switch (SW). The optical signals are then sent to the desired outputs of the switch SW. $W$-to-1 wavelength converters (WCs) are used at the SW output to converted the optical signals to the desired output wavelength channel. The wavelength multiplexer (MUX) then multiplexes the optical signals and send to the fiber for the next node. An alternate design replaces the $N W \times N W$ switch with $W N \times N$ switches working in parallel and the $W$-to- 1 WCs to 1 -to- $W$ WCs at the switch SW output [6]. We can also place the 1-to- $W$ wavelength converters at the node inputs instead as shown in Fig. 2. Although both architectures shown in Figs. 1 and 2 are functionally equivalent for the original OBS assumptions, we will show that the node architecture of Fig. 2 can be used to significantly reduce the impact of large switching overhead.

\section{B. Switching Overhead}

According to the original OBS proposed in [2], the pernode control latency of the control packet can be partitioned

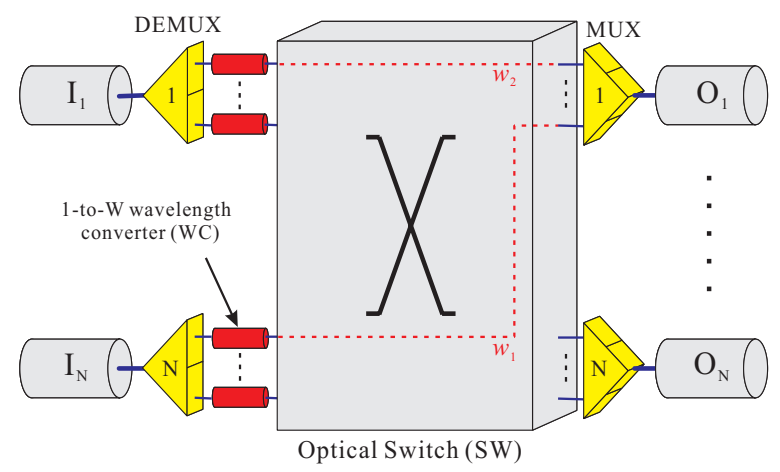

Fig. 2. An alternate design of a bufferless $N \times N$ OBS node. From left to right are the wavelength demultiplexer (DEMUX), 1-to- $W$ wavelength converters (WCs), internal non-blocking optical switch (SW), and wavelength multiplexer (MUX). The control packet processing unit is omitted.

into two parts: $T_{c p}$ the time to process the control packet and initiate the reconfiguration of hardware, and $T_{s w}$ the time required to complete the hardware reconfiguration. It has been proposed to minimize the end-to-end data burst latency by shortening the offset time between the transmissions of control packet and data burst from $H \times\left(T_{c p}+T_{s w}\right)$ to

$$
T_{\mathrm{off}}=H \times T_{c p}+T_{s w}
$$

where $H$ is the number of intermediate nodes. The idea is to overlap the hardware reconfiguration time at a node with the time for the control packet to propagate to (and possibly get processed at) the subsequent node [2]. While the switching overhead $T_{s w}$ has been separately considered in shortening the data burst latency, it has been neglected in subsequent investigations. For system throughput and data burst dropping rate computations, the difference between the per node control latency and the control packet processing time $T_{c p}$ is simply ignored [2]. This is equivalent to either neglecting the switching overhead $T_{s w}$ or treating $T_{s w}$ as part of the $T_{c p}$.

To illustrate the impact of the optical switch reconfiguration time, we assume that a control packet $C_{x}$ arrives at the node and the associated data burst $B_{x}$ will enter the node from wavelength channel $\lambda_{1}$ of input port $\mathrm{I}_{1}$ some time later. The control packet requests a wavelength channel on the desired output port $\mathrm{O}_{1}$ for data burst $B_{x}$ when it arrives at the node. Output channel selection is straightforward if the switch reconfiguration time is negligible: all channels that have sufficient idle period to accommodate the data burst $B_{x}$ are eligible, for example channel $\lambda_{2}$ of $\mathrm{O}_{1}$ in Fig. 3. If the switch reconfiguration time is not negligible, one must take into account the switch configurations before and after the switching of the data burst $B_{x}$ when selecting the output wavelength channel at an output port. For an output wavelength channel, if the switch has to reconfigure its connection in order to accept data burst $B_{x}$ and/or reconfigure to accept the data burst following data burst $B_{x}$ after $B_{x}$ exits the switch, then there must be enough time for switch reconfiguration. 


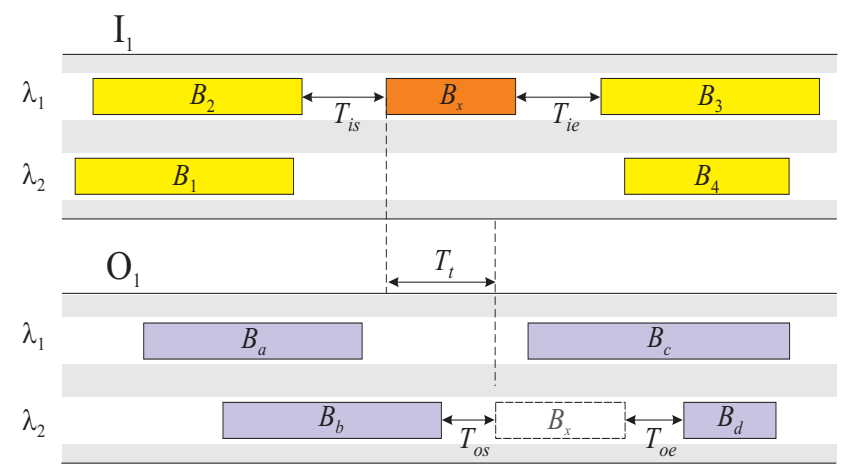

Fig. 3. Timing requirements of a data burst $B_{x}$ switched from input port $\mathrm{I}_{1}$ to output port $\mathrm{O}_{1}$ of the node shown in Fig. 1. We assume that the optical switch SW has transfer delay of $T_{t}$.

To demonstrate the performance degradation due to the switch reconfiguration overhead, we simulate OBS on an $8 \times 8$ Manhattan Street Network (MSN) [9] with the node architecture shown in Fig. 1. The assumptions and settings of the simulation have been listed in Section IV. We set the normalized offered load to be 0.2 . If the control packet processing delay $T_{c p}$ is equal to the average data burst transmission time and there is no switching overhead $\left(T_{s w}\right)$ i.e., $\left\{T_{s w}=0, T_{c p}=1\right\}$, the system has throughput of 0.15 . In Fig. 4, the solid curve is the throughput of the OBS with $T_{c p}=1$ and $T_{s w}$ varying from 0 to 0.9 . We observe that the throughput drops from 0.15 to about 0.07 when the switching overhead increases. In other words, the throughput drops by one half when the switching overhead is comparable to the control packet processing time. To illustrate the performance overestimate with the original OBS assumptions, we then assume that the switch reconfiguration time can be considered as part of the control packet processing time, i.e., the OBS now has a lengthened control packet processing time of $T_{c p}^{\prime}=$ $T_{c p}+T_{s w}$ and ignores the switch reconfiguration requirement. Assuming that each node has the same control latency, the new offset time $T_{o f f}^{\prime}$ is lengthened from that of Eq. (1) to $H \times T_{c p}^{\prime}$. Again we fix the required control packet processing time to one average data burst transmission time and vary $T_{s w}$ from 0 to 0.9 , i.e., $T_{c p}^{\prime}$ varies from 1 to 1.9 . The results are shown in the dashed curve in Fig. 4. The throughput drops slightly because the performance of OBS decreases when the average offset time increases, but the results still significantly overestimate the system performance.

To avoid the data burst dropping caused by the switching overhead, a time gap not smaller than $T_{s w}$ is in general required between data bursts. This condition can be summarized in two criteria. To simplify the illustration, we assume that when the control packet $C_{x}$ arrives at the node, data bursts $\left\{B_{2}\right.$ and $\left.B_{3}\right\}$ have already been scheduled in the input channel $\lambda_{1}$ of $I_{1}$ as shown in Fig. 3. Similarly, data bursts $\left\{B_{b}\right.$ and $\left.B_{d}\right\}$ have also been scheduled in output channel $\lambda_{2}$ of $\mathrm{O}_{1}$. We further assume that the time flow in the figure is from left to right. $T_{i s}$ and $T_{i e}$ will be the time gaps between data bursts $B_{2}$ and $B_{x}$, and data bursts $B_{x}$ and $B_{3}$, respectively. Similarly, if

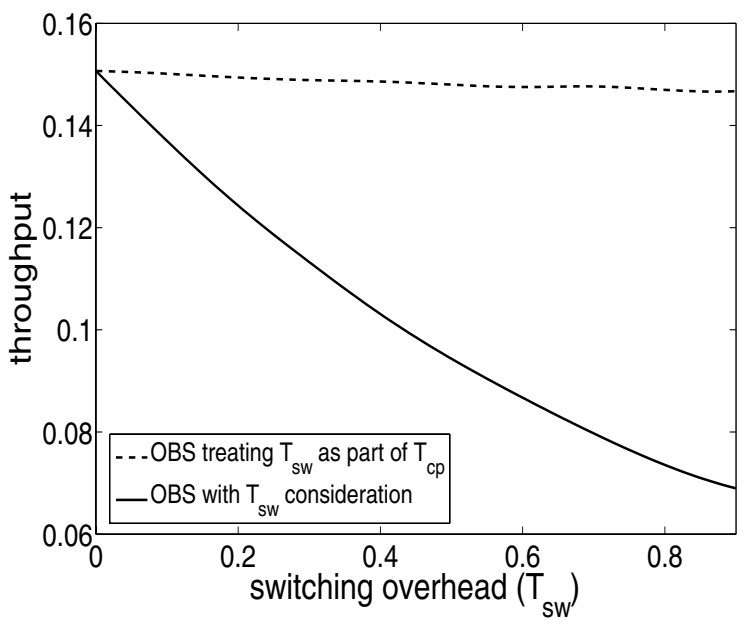

Fig. 4. The throughput of OBS with different switching overhead $\left(T_{s w}\right)$ on an $8 \times 8 \mathrm{MSN}$. The control packet processing time $\left(T_{c p}\right)$ has been set to one average data burst transmission time. The other assumptions and setting are listed in Section IV. The normalized offered load is 0.2 .

data burst $B_{x}$ is successfully scheduled, $T_{o s}$ will be the time gaps between data bursts $B_{b}$ and $B_{x}$, and $T_{o e}$ will be the time gap between data bursts $B_{x}$ and $B_{c}$. The data burst scheduling criteria are

$S_{1}$ : The scheduling is not possible if data burst $B_{b}\left(B_{d}\right)$ has been scheduled from an input port and a wavelength channel other than $\mathrm{I}_{1}$ and $\lambda_{2}$ and time gap $T_{o s}\left(T_{o e}\right)<$ $T_{s w}$.

$S_{2}$ : The scheduling is not possible if data burst $B_{2}\left(B_{3}\right)$ has been assigned to an output port and a wavelength channel other than $\mathrm{O}_{1}$ and $\lambda_{1}$ and time gap $T_{i s}\left(T_{i e}\right)<$ $T_{s w}$.

Criteria $S_{1}$ and $S_{2}$ specify the conditions that violate the reconfiguration requirement of the node switch $\mathrm{SW}$. We also know that no extra time gap will be required if input channel $\lambda_{1}$ of $\mathrm{I}_{1}$ has been connected to output channel $\lambda_{2}$ of $\mathrm{O}_{1}$ before the arrival of data burst $B_{x}$ and if the connection does not need to be torn down immediately after the transmission of data burst $B_{x}$. Criterion $S_{2}$ is not needed for a new data burst that is generated by the node itself, i.e., there is no $T_{i s}$ and $T_{i e}$ constraints. However, criterion $S_{1}$ is still applicable to the new data burst channel scheduling.

The ratio of the average time gap length to transmission time of data burst limits the maximum available system throughput. Unless the switch reconfiguration time can be reduced in proportion to the continual increase in the fiber transmission speed, the switch reconfiguration time will become increasingly significant in determining the system performance because one cannot lengthen the data burst by too much. This is a common difficulty facing all bufferless optical packetswitched networks. The OBS one way resource reservation method cannot address this issue either. 


\section{THE PROPOSED SOLUTIONS}

Owing to the resource reservation method, an OBS node can obtain from the scheduled data bursts prior knowledge of the status of the switch. This property differentiates OBS from other bufferless optical packet-switched networks, and makes it possible to reduce the impact of large switching overhead. From the discussion in Section II, devices such as the wavelength multiplexers/demultiplexers, and the wavelength converters in Figs. 1 and 2 have negligible response time when compared to that of the optical switch SW [5]-[8]. We will show in the following how to exploit the node architecture to overcome the large switching time of the optical switch SW.

\section{A. Output Channel Switching Constraint Resolution}

Criterion $S_{1}$ in Section II states that for a wavelength channel at an output port, a node need at least $T_{s w}$ time to set up a new connection from a wavelength channel of an input port different from the one currently connected. Note that the switching time $T_{s w}$ consists of two components; switching of the signal from the input to the output of the switch and conversion of the input signal from the input wavelength to the desired output wavelength. For the switch shown in Fig. 1, the optical signal is switched first and then wavelength converted. The data bursts from different wavelength channels at different input ports destined for the same wavelength channel $\lambda_{i}$ of output $O_{j}$ must use the same wavelength converter dedicated to that wavelength channel. Since that wavelength converter is connected to only one output port of the switch, the existing connection using this wavelength converter must be torn down first before a new connection can be set up. Thus the time gap between the data bursts at the output must be at least $T_{s w}$, i.e., Criterion $S_{1}$.

A simple way to overcome Criterion $S_{1}$ is to change all WCs in Fig. 1 to $W$-to- $W$ wavelength converters so that no dedicated wavelength converter and switch output port are required for an output wavelength channel. However, this becomes expensive if $W$ is large. Another method is to use the architecture in Fig. 2 instead. Since the wavelength converters are now placed at the input of the switch, in principle, we can set up a new connection to an output wavelength channel even if one connection already exists between that output wavelength channel and some input wavelength channel. For example in Fig. 2, we may set up a new connection $w_{2}$ to output wavelength channel $\lambda_{2}$ of $\mathrm{O}_{1}$ while connection $w_{1}$ already exists. Without proper control mechanism, this simultaneous connection is in general avoided to prevent data burst contention. Noted that the data bursts in the two connections are spatially separated until they reach the wavelength multiplexer. The output wavelength channel can be shared to the data burst in the two connections if we can selectively turn on one of the input wavelength converters of the two connections at any time.

Assuming that the connection $w_{1}$ has carried the data burst $B_{b}$ of Fig. 3 and connection $w_{2}$ will carry the data burst $B_{x}$, the time consuming portion of $T_{s w}$ in principle can be carried out if we can set up the connection $w_{2}$ while the data burst $B_{b}$ is still transmitting. This is possible in OBS because the resource reservation mechanism gives the node prior information of the switch SW status and channel connection requirement. The two data bursts $B_{b}$ and $B_{x}$ can therefore be placed as close as the time needed for the wavelength converters to turn off and turn on.

The performance improvement in the proposed method highly depends on the availability of idle outputs at the switch SW. In this paper, we simply assume that the number of switch outputs have been sufficiently increased so that even if all wavelength channels of an output port is occupied, a wavelength channel from an input port can still set up an internal path to the same output port to prepare for an arriving data burst.

\section{Selective Data Burst Drop}

Although the time gap between two data bursts can be much shorter than $T_{s w}$, one of the data bursts still has to be dropped if the two data bursts request different output ports at the next node because there will not be sufficient time for the switch in the next node to change the internal path, i.e., Criterion $S_{2}$. In this situation, dropping one of the two data bursts at the current node will save resources. This however does not mean that once the time gap between two data bursts is smaller than $T_{s w}$ in an intermediate node, the two data burst must use the same output ports at the nodes in the rest of their paths. This is because the two data bursts can request different wavelength channels at the same output port at one of the subsequent nodes. The two data bursts can then request different output ports in the nodes after that.

\section{B. Input Channel Switching Constraint Resolution}

There is no simple way to overcome the constraint in $S_{2}$. A hardware solution will be to double the number of 1-to$W$ wavelength converter WCs and the inputs of the switch SW in Fig. 2. The optical signal at each of the outputs of the DEMUXs is splitted into two identical parts using a $3 \mathrm{~dB}$ coupler. Let $o_{a}$ and $o_{b}$ be the DEMUX's coupler output pair for the input channel $\lambda_{1}$ of $\mathrm{I}_{1}$. Then data bursts $B_{2}, B_{x}$, and $B_{3}$ of Fig. 3 will appear simultaneously at both outputs $o_{a}$ and $o_{b}$. We assume that the time gap $T_{i s}<T_{s w}$ and data burst $B_{2}$ requests node output port $O_{N}$, not the data burst $B_{x}$ 's desired node output port $\mathrm{O}_{1}$. Since the node has prior knowledge of the switch SW status, it sets up two internal paths $P_{1}$ and $P_{2}$ in switch SW, where internal path $P_{1}$ is for data burst $B_{2}$ and internal path $P_{2}$ is for data burst $B_{x}$. When data burst $B_{2}$ arrives at the node, it is sent to the output $\mathrm{O}_{\mathrm{N}}$ by turning on the wavelength converter at $o_{a}$ and through internal path $P_{1}$ in the switch SW. At the end of the transmission of data burst $B_{2}$, the wavelength converter at $o_{a}$ is turned off and that at $o_{b}$ is turned on so that data burst $B_{x}$ is now sent to $\mathrm{O}_{1}$ using internal path $P_{2}$. In principle, the time gaps between data bursts in an input port can be close to the response time of the wavelength converters. 


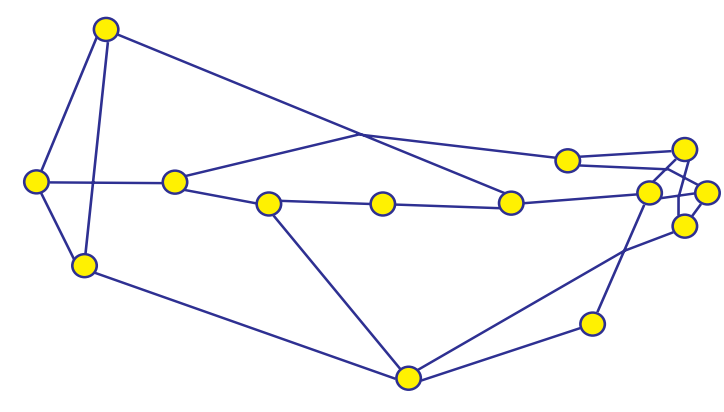

Fig. 5. The NSFNet (1991) network topology. There are 14 nodes and 21 bi-directional links. The original map of the network is available from the Internet (ftp://ftp.uu.net/inet/maps/nsfnet/) at August 2005.

\section{PERFormance EVAluation}

We use simulations to investigate the performance of the proposed node architectures and channel scheduling schemes. In the simulations, we use the network topologies of NSFNet (1991) shown in Fig. 5 and an $8 \times 8 \mathrm{MSN}$, representing irregular and regular networks, respectively. The results demonstrate that the proposed schemes can improve the performance in both kinds of networks. We focus on the system throughput performance. The traffic arrival is therefore in the form of data bursts with distribution in Poisson process. When a new data burst arrives at a node, it randomly chooses a destination from the rest of the nodes in the network and uses minimum hop routing to determine the paths. The length of each data burst (transmission time) is an exponentially distributed random variable with unit mean. The propagation delay of a link is assumed to be 62.5 time units, similar to that used in [2]. The normalized load offered to a node is the ratio of the average data burst length to the inter-arrival time normalized by the number of channels per link. In the simulations, we assume eight wavelength channels per link and all nodes receive the same offered load. All simulations are run sufficiently long such that the $95 \%$ confidence intervals are less than $1 \%$ of the results.

After a new data burst is generated at a node, a control packet is sent out immediately to reserve the required wavelength channels on the path. The data burst is then transmitted after an offset time according to Eq. (1). If two or more wavelength channels are available in a reservation, the one with smaller index is chosen. We set $T_{s w}=T_{c p}=0.5$ of the average data burst transmission time and apply the proposed schemes on both networks. For comparison, we also included the normal OBS with $\left\{T_{s w}=0.5, T_{c p}=0.5\right\}\left(\mathrm{OBS}_{0505}\right)$ and normal OBS with $\left\{T_{s w}=0, T_{c p}=1\right\}\left(\mathrm{OBS}_{0010}\right)$.

Figures 6 and 7 show the throughput of the proposed solutions in Section III under different offered loads and network topologies. The curves with plus, asterisks, and squares are the throughput for the schemes of OBS with output channel switching constraint resolution but no selective data burst drop (OCS), OBS with output channel switching constraint resolution and selective data burst drop (OCS/SD), and OBS with both input and output channel switching constraint resolution

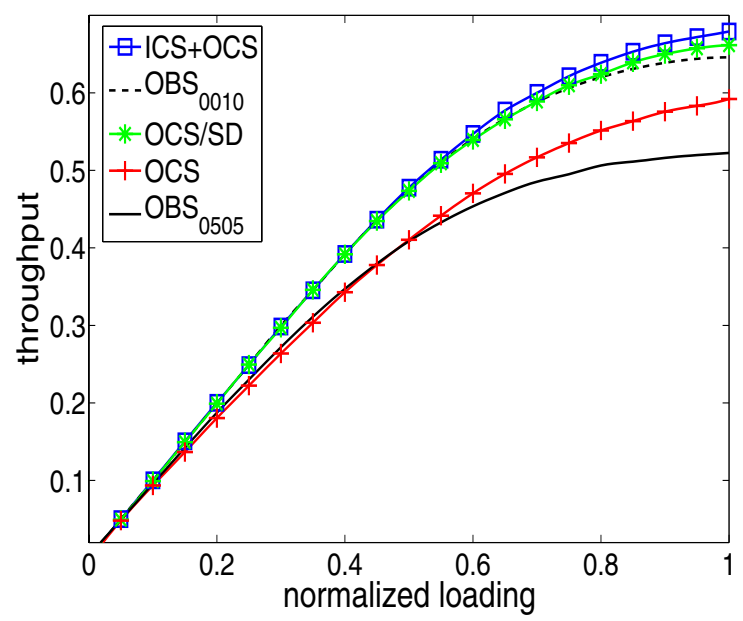

Fig. 6. The throughput of the proposed switching constraint resolution schemes on the NSFNet topology (shown in Fig. 5) network with $T_{s w}=$ $T_{c p}=0.5$, where OCS: OBS with output channel switching constraint resolution but no selective data burst drop, OCS/SD: OBS with output channel switching constraint resolution and selective data burst drop (Section IIIA), ICS+OCS: OBS with both input and output channel switching constraint resolution (Sections III-A and III-B), OBS 0505 : normal OBS with $\left\{T_{s w}=\right.$ $\left.0.5, T_{c p}=0.5\right\}$, and OBS 0010 : normal OBS with $\left\{T_{s w}=0.0, T_{c p}=1.0\right\}$

(ICS+OCS), respectively. The solid and dashed curves are the throughput for the normal OBS with $\left\{T_{s w}=0.5, T_{c p}=0.5\right\}$ $\left(\mathrm{OBS}_{0505}\right)$ and normal OBS with $\left\{T_{s w}=0, T_{c p}=1\right\}$ $\left(\mathrm{OBS}_{0010}\right)$, respectively. In the simulations, all throughput curves with the NSFNet increase monotonically but those with $8 \times 8 \mathrm{MSN}$ do not. Fig. 7 shows the throughput curves for $8 \times 8 \mathrm{MSN}$ start to drop when the offered load exceeds 0.2 . It is because the average and maximum path lengths of NSFNet are 2.148 and 3 hops, respectively, while that of an $8 \times 8 \mathrm{MSN}$ are 5.016 and 9 hops, respectively. Hence, a data burst on average passes one intermediate node in the NSFNet topology network but encounters 4 in the $8 \times 8 \mathrm{MSN}$. Moreover, each link in the NSFNet topology network supports on average 9.308 sourcedestination pairs while that of the $8 \times 8$ MSN supports 158 . Congestion is more likely to occur in the $8 \times 8 \mathrm{MSN}$ than in the NSFNet topology network.

From Figs. 6 and 7, we find that the normal OBS with $\left\{T_{s w}=0.5, T_{c p}=0.5\right\}$ has the worst throughput performance. The throughput of OBS with both output and input channel switching constraint resolution (ICS+OCS) is slightly better than the normal OBS with $\left\{T_{s w}=0, T_{c p}=1\right\}$ and also better than all the rest. Because of its longest average offset time, the normal OBS with $\left\{T_{s w}=0, T_{c p}=1\right\}\left(\mathrm{OBS}_{0010}\right)$ has throughput slightly lower than that of ICS+OCS (the OBS with both output and input channel switching constraint resolution). In general, OBS with smaller offset time has better throughput.

The throughput performance of the OCS/SD (OBS with output channel switching constraint resolution and selective data burst drop) in Fig. 6 is similar to that of the normal OBS with $\left\{T_{s w}=0, T_{c p}=1.0\right\}\left(\mathrm{OBS}_{0010}\right)$ but the throughput 


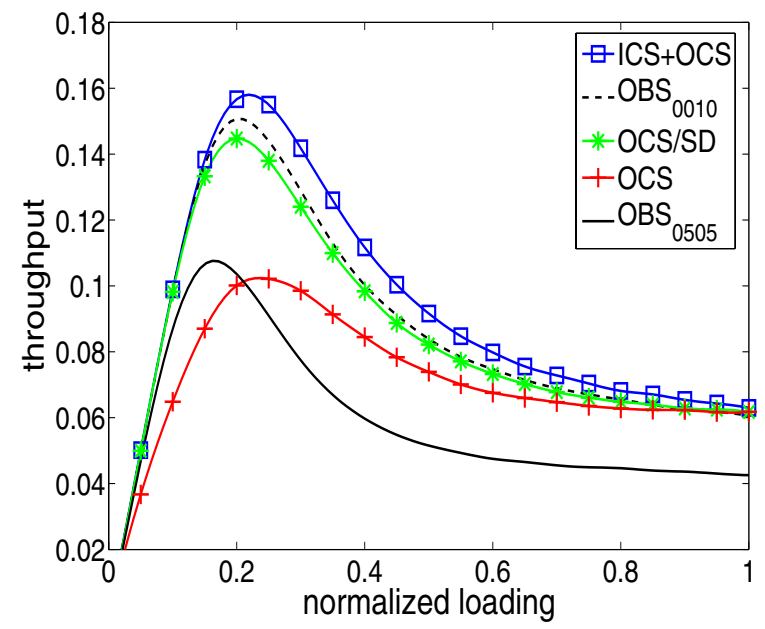

Fig. 7. The throughput of the proposed switching constraint resolution schemes on an $8 \times 8 \mathrm{MSN}$ network with $T_{s w}=T_{c p}=0.5$. The notations are similar to that in Fig. 6.

performance of the OCS/SD in Fig. 7 is slightly lower than that of $\mathrm{OBS}_{0010}$. The difference in the performance of OCS/SD in the two cases is due to the NSFNet having a lower average path length and smaller traffic intensity on each link when compared to that of the $8 \times 8 \mathrm{MSN}$. We observe that the effect of input switching constraint is not significant in the NSFNet but is not negligible in the $8 \times 8 \mathrm{MSN}$.

From Figs. 6 and 7 the throughput performance of the OCS (OBS with output channel switching constraint resolution but no selective data burst drop) is lower than that of the normal OBS with $\left\{T_{s w}=0, T_{c p}=1.0\right\}\left(\mathrm{OBS}_{0010}\right)$ and OCS/SD. We observe a significant drop in the throughput performance if the selective data burst drop is not used. In Fig. 7, the OCS has a lower throughput than the $\mathrm{OBS}_{0505}$ when the offered load is between 0 and 0.2 . The throughput of OCS converges to that of $\mathrm{OBS}_{0010}$ and ICS+OCS at the high loading range because single hop traffic dominates the network in the high loading situation such that there is no significant difference between OCS, OCS/SD, ICS+OCS and OBS 0010 .

\section{CONCLUSION}

In this paper, we review the impact of switching overhead on the performance of optical burst switching (OBS) systems. As the optical fiber transmission rate exceeds hundreds gigabits per second, the switching overhead will become a significant problem. In the past, people treat the switching overhead as part of the control packet processing delay and designed the OBS routing and resource reservation schemes accordingly. This will largely overestimate the OBS system performance in large switching overhead situations. Because of its resource reservation method, an OBS node can have prior knowledge of the status of the switch which is not available in other kinds of optical packet-switched networks. We use the information to alleviate the negative impact on the system performance of large switching overhead.

\section{REFERENCES}

[1] J.S. Tuner, "Terabit burst switching," Journal of High Speed Networks, Vol. 8, pp. 3-16, 1999.

[2] C. Qiao, and M. Yoo, "Optical burst switching (OBS) - a new paradigm for an optical Internet," Journal of High Speed Networks, Vol. 8, pp. 6984, 1999.

[3] J.Y. Wei, and R.I. McFarland, Jr., "Just-In-Time Signaling for WDM Optical Burst Switching Networks," Journal of Lightwave Technology, Vol. 18, No. 12, pp. 2019-2037, 2000.

[4] Y. Sun, T. Hashiguchi, N. Yoshida, X. Wang, H. Morikawa, and T. Aoyama, "Architecture and design issues of an optical burst switched network testbed," Proceedings of OECC/COIN 2004, pp. 386-387, 2004.

[5] X.H. Ma, and G.S. Kuo, "Optical switching technology comparison: optical MEMS vs. other technologies," IEEE Communications Magazine, Vol. 41, No. 11, pp. S16-S23, 2003.

[6] G.I. Papadimitriou, C. Papazoglou, and A.S. Pomportsis, "Optical Switching: Switch Fabrics, Techniques, and Architectures," Journal of lightwave Technology, Vol. 21, No. 2, pp. 384-405, 2003.

[7] J.M.H. Elmirghani, and H.T. Mouftah, "All-optical wavelength conversion: technologies and applications in DWDM networks" IEEE Communications Magazine, Vol. 38, No. 3, pp. 86-92, 2000.

[8] I. White, R. Penty, M. Webster, J.C. Yew, A. Wonfor, and S. Shahkooh, "Wavelength switching components for future photonic networks," IEEE Communications Magazine, Vol. 40, No. 9, pp. 74-81, 2002.

[9] N.F. Maxemchuk, "Routing in Manhattan Street network," IEEE Transactions on Communications, Vol. 35, No. 5, pp. 503-512, 1987. 